\title{
Computational tools for evaluating bioemergency contingency plans
}

\author{
T. Schneider, A. R. Mikler \& M. O’Neill II \\ Center for Computational Epidemiology and Response Analysis, \\ University of North Texas, USA
}

\begin{abstract}
Bioemergencies and bioterrorism play an increasingly important role in public health disaster preparedness. Posing a major challenge, the timely distribution of vaccine and medication to the population in case of an emergency requires contingency plans to be thoroughly analyzed. The enormous scale of possible response scenarios together with intrinsic confidential requirements make field studies both infeasible and unpractical. Computational tools offer the ability to evaluate different scenarios and alter contingency plans instantaneously. Here, we describe the methodology for the evaluation of response scenarios involving the dispension of vaccine or medication at fixed locations within a geographic region.

Keywords: pandemics and biological threats, terrorism, emergency preparedness and planning, disaster resilient communities, public health preparedness.
\end{abstract}

\section{Introduction}

County health departments in the US have received federal and state funds to develop response plans for biological and health emergencies that are naturally occurring or the result of bioterrorism. Depending on the type of the biological threat, health departments must be prepared to respond by coordinated intervention within a time frame mandated by the Centers for Disease Control and Prevention (CDC).This has led to the designation of disaster preparedness committees, who have identified points of distribution (PODs), at which in the event of such an emergency the population of the county can be supplied with vaccines and/or prophylactic medication. However, assessing the feasibility of such a contingency plan in general, and the placement of PODs in particular, constitutes a significant challenge, as it requires that demographic data, traffic information, geographic 
layout, and POD capacities be taken into consideration. We have developed computational tools that will aid the planning and assessment process and facilitate immediate re-allocation of public health resources. This paper will highlight the methodologies that have been developed to predict whether the existing road network can support a specific POD placement and facilitate a county's emergency response strategies. Associated with the PODs are specific catchment areas determining the expected demand for each of the PODs. A corresponding road model with base traffic, speed limits, number of lanes, and traffic counts allows for the estimation of traffic feeding into each POD. The design of a feasible contingency plan represents a multi-variate optimization problem that necessitates the use of computational tools. These tools must be general enough to facilitate the planning and validation process in different locations for different types of bioemergencies and geographic locations and must be designed to be usable by policy makers and public health experts.

\section{Related work}

Throughout history, diseases have threatened the world's population. While historically, naturally occurring events were of major concern, disaster preparedness committees are now forced to also incorporate contingency plans for bioemergencies inflicted by terrorists. The authors in [1] compare the public health officials as first responders for bioterrorist attacks to first responders for conventional terrorist attacks, such as policemen, firefighters, and paramedics. In [2], the authors claim that "any small or large outbreak of disease should be evaluated as a potential bioterrorist attack". Recent events have shown that a public health event automatically raises human suspicion, which became evident, when an Australian scientist suggested that the swine flu was not naturally occurring, but produced in a lab. The WHO, however, disagreed [3]. In recent years, people have become increasingly aware of the fact that in order to generate good solutions for such complex problems, interdisciplinary collaboration is important. In [4] the importance of this collaboration is stressed, while indicating that public health itself is an interdisciplinary field. For early warning systems to be successfully implemented, clinicians must work closely together with public health officials [5]. Not only the need for collaboration, but also the necessity of having effective disease surveillance in place, is emphasized in [6]. Additionally, a distinction between naturally occurring disease outbreaks and bioterrorist attacks must be carefully evaluated using disease characteristics with respect to geographic area, season, and mode of transmission.

The use of GIS for public health events is not a novel approach. A well-known example is John Snow's famous cholera map of London published in 1855 [7], which helped to identify a specific water pump as the cause for the outbreak. In the past decades, however, the use of GIS in public health and epidemiology has experienced a revival, making use of geographic information to improve public health. A field study was conducted during a disaster preparedness exercise in Montana with an emphasis on demonstrating the usefulness of GIS integration 
into emergency planning [8]. In North Carolina, GIS is already being deployed, whereby the mobile GIS software ArcPad replaces the use of paper forms in order to speed disaster relief [9]. Assessment teams use it on mobile devices to calculate routes to survey locations and to facilitate the completeness of data entries. The collected data can be directly uploaded into a database and therefore, eliminating data re-entry.

Databases filled with public health and geographic data are being maintained at public health authorities, and as early as in 1985 the role of the use of Computer Science in the domain of public health has been acknowledged [10]. Now, computational models are ubiquitous, modelling different diseases to support public health officials in the development of intervention strategies, as demonstrated for HPV in [11]. More recently, data mining of social media has been used to show strong correlation between the Morbidity and Mortality Weekly Report (MMWR) by the CDC and the occurrence of words related to influenza in blog posts [12].

\section{Methodology}

Given the POD locations, census data, road network information and traffic statistics, models have been developed aiding public health officials to evaluate and adapt contingency plans. This section outlines the steps necessary to build a framework for such an analysis for a given geographic location.

\subsection{Defining catchment areas}

Based on existing POD locations, a county is divided into catchment areas. Each of the catchment areas is pertinent to a single POD and defines the population that the corresponding POD must serve. Using census block granularity, each census block is collapsed into its geographic centroid, which allows for its representation by a single point. Making use of these centroid locations, the distances between the centroids $C$ and PODS $P$ are calculated and a centroid $c \in C$ representing its corresponding census block is assigned to a POD $p \in P$, if the distance between $c$ and $p$ is smaller than the distance between $c$ and any of the other PODs $p^{\prime} \in P \backslash p$. This assignment can be expressed as follows:

$$
\forall c \in C: \operatorname{pod}(c)=\min (\|c-p\|)
$$

where $\operatorname{pod}(c)$ takes a census block as an argument and returns the corresponding POD. As a first approach, the Euclidean distance is used as the distance metric, although other metrics, such as minimum road distance, minimum travel time, and a weighted combination of different metrics can be considered in order to account for various factors, including natural and unnatural boundaries (rivers, lakes, mountains, walls, ...). The resulting partitioning of the county resembles a Voronoi decomposition, adjusted to be delineated by census block borders. This approach is depicted in Figure 1. Figure 1(a) shows census blocks with their corresponding centroids. The POD locations are marked by triangles. In Figure 1(b) the 


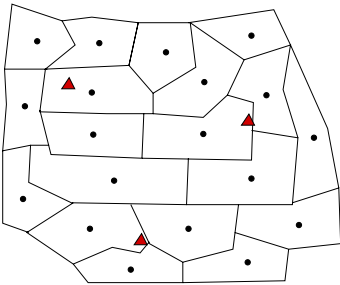

(a) Census blocks and PODs

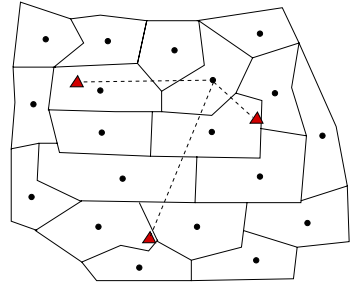

(b) Distance to all PODs

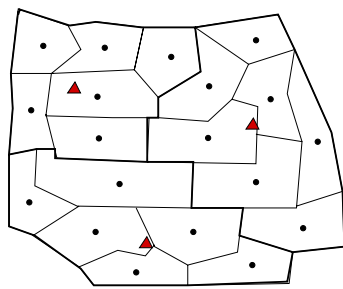

(c) Catchment areas

Figure 1: Determining catchment areas.

process of finding the closest POD is illustrated, which leads to the partitioning of the geographic space into catchment areas, as shown in Figure 1(c).

Note that the described approach is not limited to census block granularity, but can be applied to different geographic entities. However, using census blocks allows for estimating the population count within a catchment area with a relatively fine granularity. By dividing a county into catchment areas, we have reduced the problem of analyzing a geographic space with multiple PODs to the task of evaluating a region allocated to a single POD. The traffic intensity is likely to increase towards the PODs with the highest traffic volume close to the POD location, and less traffic around the catchment area borders. In the following sections, we describe how we make use of this property in order to estimate traffic demands.

\subsection{Including the road network}

Assuming different traffic densities as a function of distance, the POD can be represented by concentric rings surrounding the POD. Let $k$ denote the number of rings and $d_{\max }$ the point in the catchment area that is furthest away from the POD, then the census blocks of a catchment area pertinent to a POD $p$ are classified into sets of rings as follows:

$$
R_{i}=\left\{c \in C \mid(i-1) \frac{d_{\max }}{k}<\|c-p\| \leq \frac{d_{\max }}{k}\right\}
$$

where $i \in\{1 \ldots k\}$. Note that census block boundaries are always respected and therefore, the rings are conforming to these boundaries. An example of such a division into rings with $k=3$ is illustrated in Figure 2. In Figure 2(a) the catchment area with its census blocks and POD is shown. The resulting subdivision after the ring assignment is depicted in Figure 2(b). It is obvious, that in order to travel to the POD from ring $R_{i}$ with $i \in\{1 . . k\}$, all $R_{j}$ with $j \in\{1 . . i\}$ have to be traversed. This is illustrated in Figure 2(e).

Once the rings are computed, road network information is superimposed with the amount of detail obtainable. Hence, minor roads might not be considered, which does not represent a limitation to the model, as these roads mainly contribute to local neighborhood traffic. For each of the rings, the intersection points 


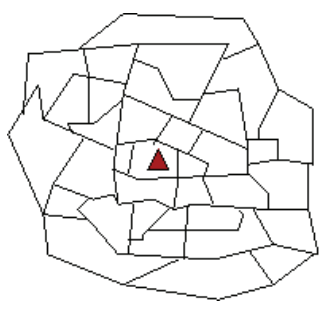

(a) Catchment area for one POD

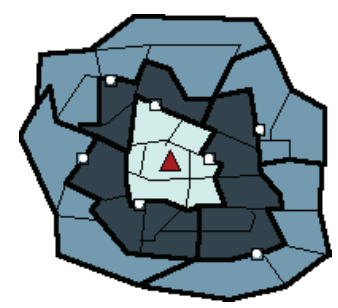

(d) Subsectors

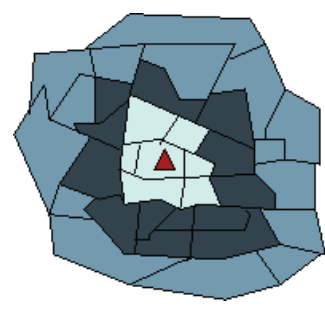

(b) Concentric rings around POD

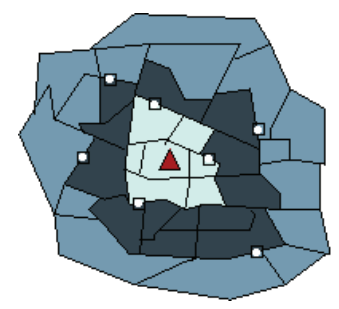

(c) Crossing points

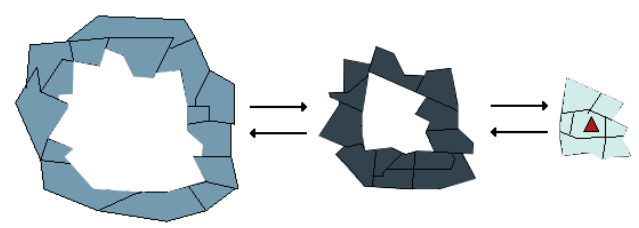

(e) Traffic flow graph

Figure 2: Subdividing catchment areas.

with the road network are computed (Figure 2(c)). These points can be considered bottlenecks, as in order to reach ring $i-1$ from ring $i$, they must be traversed. Hence, these points provide a measure of traffic influx into the next inner ring. The actual number of cars traversing these crossing points depends on the number of people living in the census blocks of the rings, and the base traffic pertinent to the surrounding areas. The latter will be addressed in the next section.

To determine the influx for each of the individual crossing points, the rings are further subdivided. Analogous to the determination of the catchment areas, all census blocks of a ring are assigned to the closest crossing point of ring $i-1$ (Figure 2(d)). It should be noted that there is no subdivision for the inner ring $R_{1}$, as it contains the POD itself. Starting with the outermost ring $R_{k}$ the population of each subsector is added to obtain an estimate of the number of people crossing into the next ring $R_{k-1}$. Parameters, such as the average number of people per car, can be specified by public health officials to compare different assumptions and evaluate the resulting scenarios. Once the traffic feeding into ring $i-1$ is estimated, the crossing point is assigned this number and acts as a "super-centroid" for the next iteration. The closer the rings are to the POD location, the higher the expected traffic feeding into the crossing points. This conforms with the scenario we would expect in the case of a real emergency.

The expected scenarios, however, are likely to differ depending on which time of the day the emergency is announced. At night the population is assumed to conform with the spatial distribution as obtained from the census data. Nevertheless, 
during daytime, parts of the population are not in the census block they live in, due to work, school, and other activities. Also, in city areas, one has to account for additional population of the workforce from surrounding areas, who might not live in the affected county. Hence, the time of the day of the announcement can play a crucial role in the results of the analysis.

\subsection{Modelling traffic flow}

Once the expected distribution of the population is calculated, the base traffic can be estimated and road network information can be integrated into the model. The available information includes traffic counts, the number of lanes, and speed limits. Traffic counts, however, are only available for a minor amount of the road segments of the data set. Hence, traffic information must be interpolated for those segments, for which no traffic counts are available. As mentioned above, some minor roads are not included in the infrastructure description, and can consequently not be considered as part of the road network. Traffic counts for major roads are available in 15 minute intervals.

The methodology to estimate traffic counts is sufficiently generic to be applicable to all roads in the road network. In the following we are assuming the average length of a car $l_{c}$ to be 6 meters feet, the space between two cars $l_{s}$ to be 1 car length per $16 \mathrm{~km} / \mathrm{h}$, and given the speed limit $v_{\max }$ and the number of lanes $n$, the maximum capacities $C_{\max }$ for a road segment can be calculated as

$$
C_{\max }=\frac{v_{\max }}{l_{s}+l_{c}}=\frac{v_{\max }}{\left(\frac{v_{\max }}{16}+1\right) \times 6 \times 10^{-3} \mathrm{~km}}
$$

$C_{\max }$ is a measure of how many cars can at most traverse a road segment. Using the actual traffic counts we calculated the proportion of the maximum traffic capacities. This was done for different times of the day and allows us to estimate the roadway traffic for any road segment with the given parameters.

Figure 3 shows the traffic count distributions over a day comparing proportions of total daily counts for mid-week and weekend traffic. Specific traffic counts have been obtained from the local department of transportation. Taking into account that we consider different road segments and different dates, the standard deviation is within reasonable limits. Comparing the graphs for the different days of the week, two patterns have been observed that lead to the formulation of two distinct traffic distribution classes, namely for weekdays and weekend days. The two classes are shown in Figure 4. For both scenarios, a very small standard deviation can be observed. The graphs represent the actual proportion $P_{c}(t)$ of traffic counts averaged over all road segments with available traffic counts for a given time interval, which are calculated as

$$
P(t)=\frac{1}{|S|} \sum_{s \in S} \frac{C_{\text {actual }}^{s}}{C_{\max }^{s}}
$$

where $S$ is the set of all road segments with available traffic counts. 


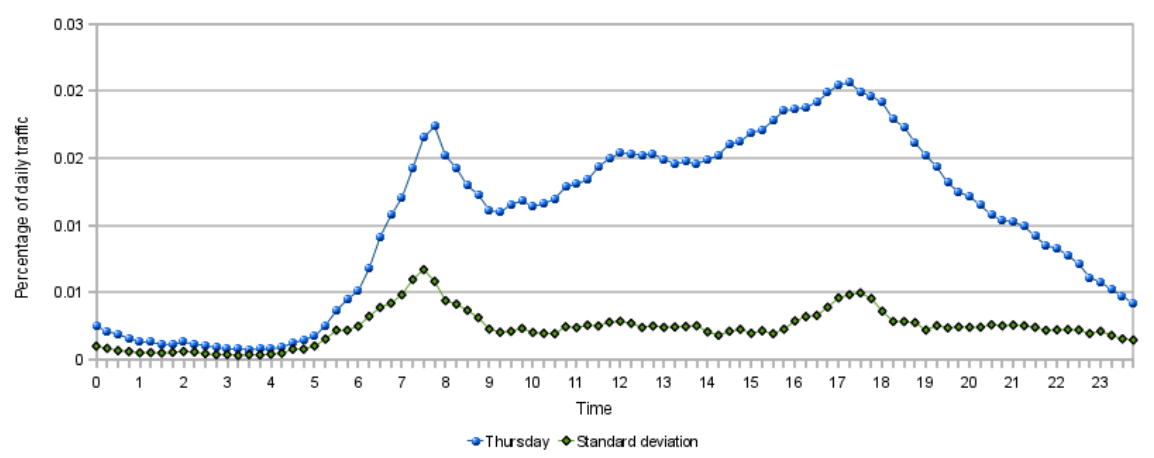

(a) Traffic counts for Thursdays

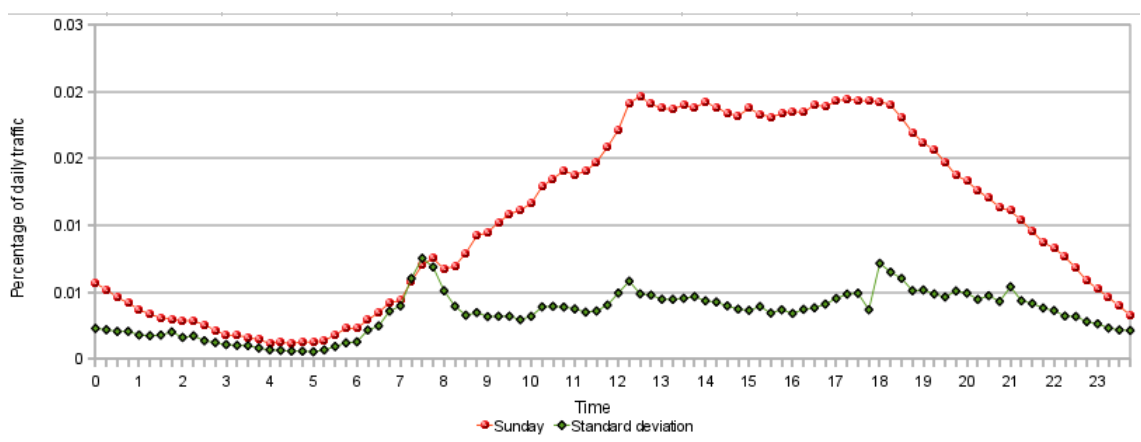

(b) Traffic counts for Sundays

Figure 3: Traffic counts for a sample weekday and weekend day across all different road segments with available counts.

Elaborate models of how to predict future traffic have been created and estimates for the future are given based on current and historical data [13]. Also techniques for estimating traffic data from single-loop or dual-loop detectors have been developed [14]. The Joint Research Center of the European Union published a document discussing collection methods and applications of road traffic data, in which for different European and US locations, traffic graphs for weekdays are shown [15]. Interestingly, the graphs exhibit the same general shape as depicted in Figure 4(b).

\subsection{Traffic along the borders of the catchment areas}

Although we assume most of the traffic to occur close to the POD locations and away from the catchment area borders, we have to account for some cross-border traffic. There are two cases that have to be distinguished: Either a catchment area border shares its border with another catchment area, or it borders with a neighboring county. In the first case we assume an overlap of width $w$. This allows for modeling the traffic around the border, simulating that people living in areas with 
40 Disaster Management and Human Health Risk

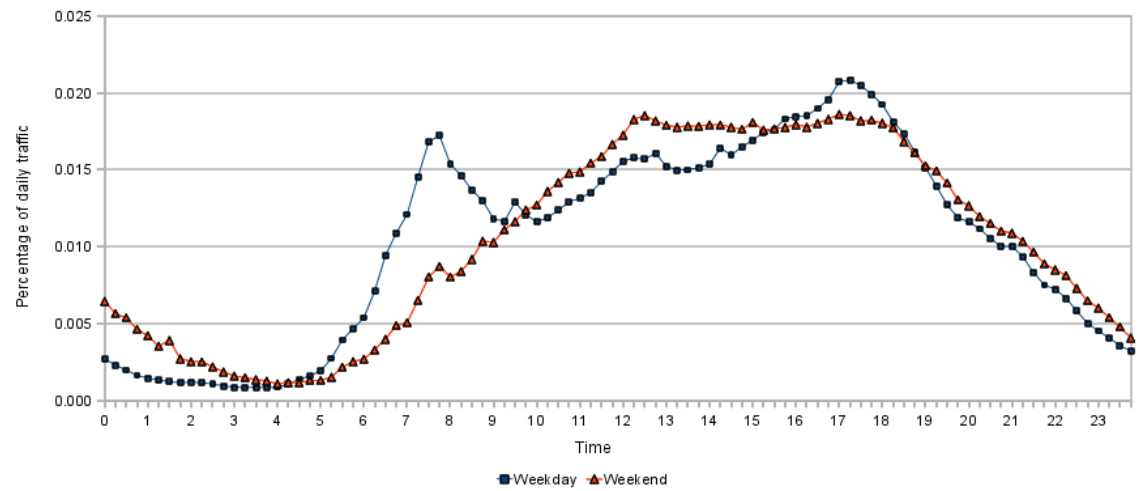

(a) Weekdays and weekend

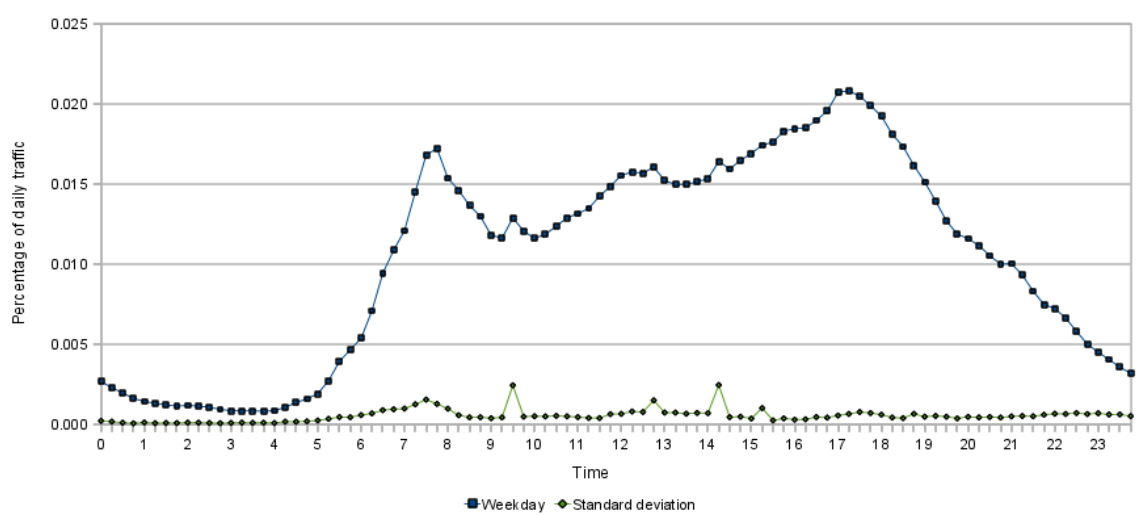

(b) Weekdays

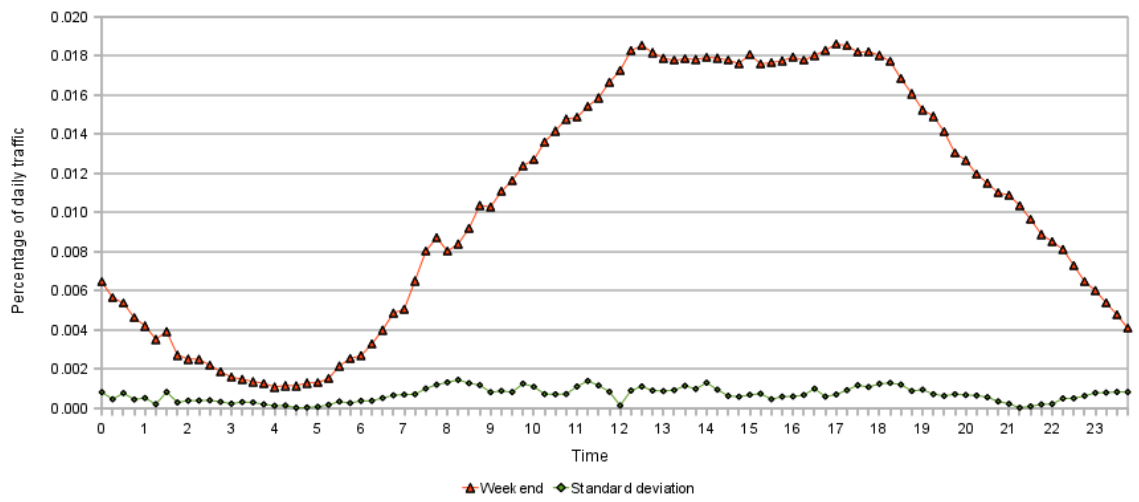

(c) Weekend

Figure 4: Traffic classes. 


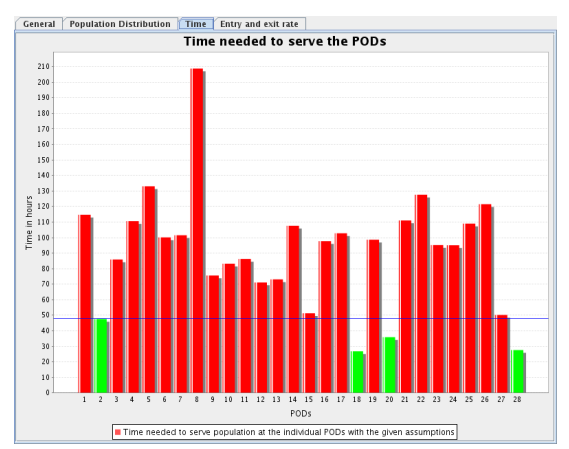

(a) Estimated serving time

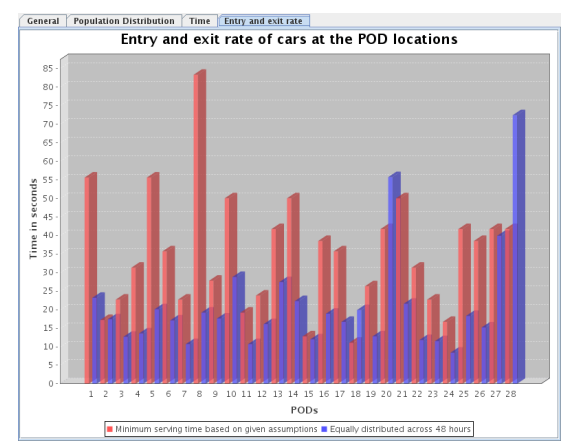

(b) Entry and exit rates

Figure 5: PODAnalyzer.

more than one POD at a similar distance might choose any one of the PODs. For catchment areas sharing borders with neighboring counties, additional incoming traffic must be anticipated. If the neighboring county is following similar procedures at the same time, the border traffic is treated similar to the situation of shared catchment areas. However, if neighboring counties do not provide public health services, the model needs to account for additional traffic. Further, it has been observed that people tend to show more panic in case of unnatural disasters, which can lead to an increase of the population to be served [16]. For instance, during a chemical spill in Arkansas, an additional 32\% decided to evacuate, although they were instructed to seek shelter. Instances like this indicate that additional people from the surrounding counties might enter the county to obtain vaccines or medication, although their area of residence is not affected.

\subsection{Feasibility at the POD locations}

Given that the road network can support the anticipated traffic, the feasibility of the contingency plan at the POD locations themselves must be verified. A tool has been developed that allows public health officials to specify parameters, such as people per car, estimated serving time per car, number of booths per POD location, and determines whether the population of a catchment area can be served within a mandated time interval. The length of this interval varies with the type of the underlying emergency, which will determine whether individuals are vaccinated or merely provided medication. The interface of the tool is depicted in Figure 5, showing estimated time requirements for each POD to serve its catchment area, and the estimated and optimal entry and exit rates for cars into and out of the POD locations. 


\section{Summary}

This paper briefly summarizes the methodology that has been developed to assess the feasibility of specific responses to possible bioemergencies. In particular, computational tools have been designed to gage the throughput of dispensing points, at which the public can obtain vaccines or prophylactic medication. An integral part of this analysis is the estimation of anticipated traffic in the road network during a bioemergency, as this can drastically impede access to the PODs. In general, computational models and tools offer public health officials means to evaluate the feasibility and effectiveness of bioemergency contingency plans. Not only do they offer functionality for finding adequate strategies responding to possible biothreats, but they can also facilitate the reallocation of resources in response to emerging conditions. For instance, in the event of a POD location being compromised, computer tools can quickly adapt the original strategy to new circumstances. Often, data pertinent to such contingency plans is strictly confidential, including POD locations and exact procedures and hence, field exercises cannot be conducted. The analytical study of response scenarios constitutes a complex optimization problem that necessitates the development of new computational tools.

\section{References}

[1] Shalala, D., Bioterrorism: How prepared are we? Emerging Infectious Diseases, 5(4), 1999.

[2] Pavlin, J., Epidemiology of bioterrorism. Emerging Infectious Diseases, 5(4), 1999.

[3] McNeil, D., Swine flu not an accident from a lab, w.h.o. says. New York Times, 2009.

[4] Shoaf, K. \& Rottman, S., The role of public health in disaster preparedness, mitigation, response, and recovery. Prehospital and Disaster Medicine, 2000.

[5] Gerberding, J., Hughes, J. \& Koplan, J., Bioterrorims preparedness and response: Clinicians and public health agenscies as essential partners. JAMA, 287(7), 2002.

[6] McDade, J. \& Franz, D., Bioterrorism as a public health threat. Emerging Infectious Diseases, 4(3), 1998.

[7] Snow, J., On the mode of communication of cholera. J Churchill, 1855.

[8] ESRI, Disaster preparedness exercise uses gis. ESCRI ArcNews, 28(1), 2006.

[9] ESRI, Mobile gis speeds disaster relief. ESRI, 2005.

[10] Hunt, A., Morrison, P. \& Pagano, M., The effects of computer science advancements on public health research. Annual Review of Public Health, 6, 1985.

[11] Corley, C., Brown, L., Mikler, A., Cook, D. \& Singh, K., Generating social networks of intimate contacts for the study of public health intervention strategies. Proceedings of the IEEE 7th International Symposiums on Bioinformatics and Bioengineering, 2007. 
[12] Corley, C., Mikler, A., Singh, K. \& Cook, D., Monitoring influenza trends through mining social media. Proceedings of the 2009 International Conference on Bioinformatics and Computational Biology, 2009.

[13] Wang, X. \& Kockelman, K., Forecasting network data: Spatial interpolation of traffic counts using texas data. Transportation Research Record, 2009.

[14] Rakha, H. \& Zhang, W., Estimating traffic stream space mean speed and reliability from dual-and single-loop detectors. Transportation Research Record, 2005.

[15] Leduc, G., Road traffic data: Collection methods and applications. JRC Technical Notes, 2008.

[16] Saathoff, G.B. \& Jr., J.B.N., Mass evacuation and our nation's highways. Carolina Panning, 2005. 